\title{
Design, development, application, safety assessment and simulation of the railway signaling system
}

\author{
B. Ning, T. Tang, C. Gao \& J. Xun \\ The State Key Laboratory of Rail Traffic Control and Safety, \\ Beijing Jiaotong University, P.R. China
}

\begin{abstract}
The railway signaling system is one of the key subsystems in railway systems to ensure the train operation efficiency and safety. It is a complicated system. However, the railway signaling system is not independent in railway systems. In this paper, five parts of the railway signaling system with their features and the relationship are described in detail. Firstly, the core system of the railway signaling system is designed and developed. Re-design is carried out for the application of the core system for the specified rolling stocks and lines. The safety of the core system and the applied system needs to be assessed. Finally, a complete simulation system should be built for testing, installation, maintenance and the technique upgrading of the systems. This paper helps people to get a deep understanding about the functions, design and development, applications and simulation of railway signaling systems.
\end{abstract}

Keywords: railway signaling system, system design, safety assessment, simulation.

\section{Introduction}

The railway signaling system is the brain and nerve system of railway systems, which ensures the safety and efficiency of the train operation. However, compared with civil engineering, such as lines, bridges, tunnels, and rolling stock, the cost for a signaling system is relatively low. Generally speaking, it is less than $10 \%$ of the whole cost for a railway system. Little attention has been paid give to it, either for the main line railway systems or the underground ones. 
With the quick development of railway systems, especially in the high-speed railways and high-density urban transit systems, the importance of the signaling system has been realized by more and more people. In order to get a better understanding of the railway signaling system, we divide it into the core system, the minimal system and the application system, according to their functions and applications. Meanwhile, design, re-design, simulation, and safety assessment of the railway signaling system in particular are also introduced.

There are two typical railway control systems in the world, which have been developed into standardizations. One is the ETCS (European Train Control System) for the railway signaling system in Europe, the other is the CTCS (Chinese Train Control System) for the railway signaling system in China. In this paper, the two systems are taken as examples to show how the signaling systems are designed, developed, re-designed, assessed and simulated. The core systems of a railway signaling system are defined. According to the requirements of the application, the task of the core systems is described. The railway signaling system is a requirements-tailored product for different lines and different rolling stocks. Furthermore, the railway signaling system must be fail-safe and reliable. In the design of the core system and the re-design of an applicable system, some of the special principles must be considered. Therefore, safety assessment must be carried out for the signaling system. In addition, the simulation system has become one of the necessary tools for the design, application and maintenance of the signaling system.

Much knowledge is accumulated during the whole cycle of the signaling system, while it is relatively simple from the view of the function points. With the introduction in the following sections, people will understand why the railway signaling system is important, special and high cost.

\section{Definition of railway control systems and their core systems}

The railway system can generally be divided into three parts shown in Fig. 1.

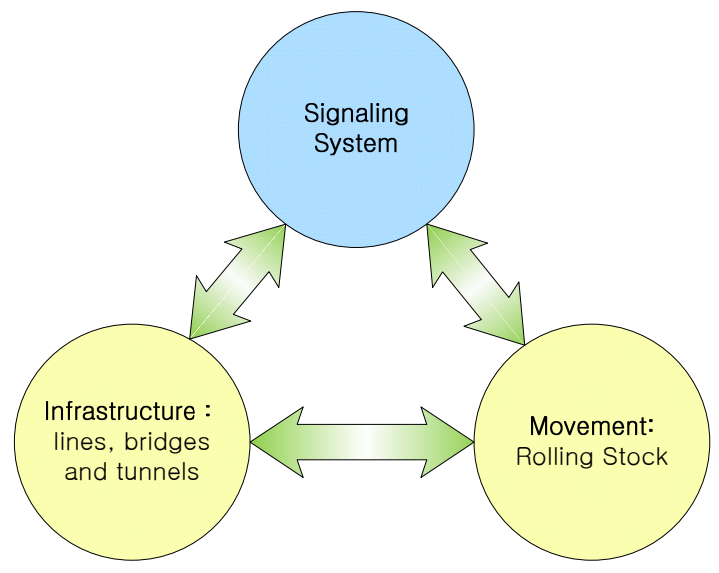

Figure 1: The railway system. 
One part is the infrastructure, which includes the lines, bridges, and tunnels. It is called the fixed part of railway Fsystem. The second part is the rolling stock, which is called as the movement part of railway system. The third part is the signaling system, which is called the brain and the nerve system of railway system.

As shown in Fig. 1, trains run on the lines controlled by the signaling system in any railway systems. Therefore, the signaling system ensures trains to operate safely and efficiently. The roles and functions of the signaling system in railway systems are clearly stated in Fig. 1. It is obvious the signaling system is the brain and the nerve system of railway system. Without signaling system, railway systems cannot operate efficiently and safely. It also can be seen that the signaling system has close relationship with rolling stocks and infrastructures.

The configuration of the signaling system is given in Fig. 2. Usually, there are four parts included in the signaling system: (1) On-board control system, (2) Station control system and wayside system, (3) Central control system, (4) Communication network including mobile telecommunication.

The core systems of the signaling system are consisted by the above four parts in Fig. 2. The interlocking system and RBC (Radio Block Control) belong to the station control systems. The on-board control system, control center and the communication system are also one of the core systems for signaling system. In more details, the vital computer for interlocking system, on-board system and $\mathrm{RBC}$ system, and the basic software for the four parts are also belong to the core part of the signaling system. In the paper, the core systems are the foundation of the signaling system, and are called as the basic models of the signaling system.

Up to now, the functions of the signaling system in railway system, and the relationship between the core systems and the signaling system are explained. When ETCS or CTCS is analyzed, the four parts, or the core systems can be seen easily. In the ETCS, there are Euro-cab, Euro-radio (GSM-R) and Eurointerlocking, etc. In the CTCS, there are Chinese on-board system (Universal cab signaling), GSM-R and Chinese interlocking systems (four kinds of interlocking systems), etc. as in Ning et al. [3].

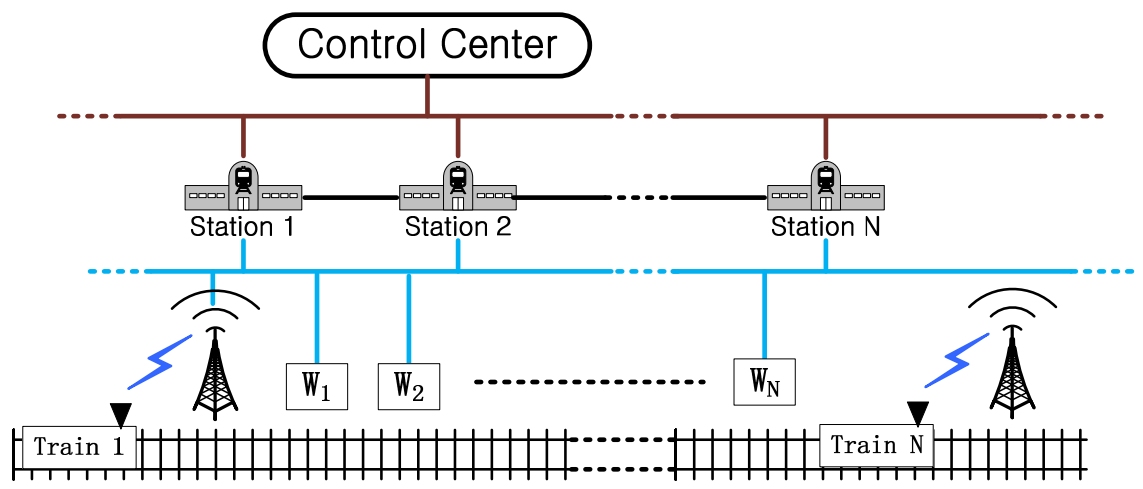

Figure 2: $\quad$ Configuration of railway signaling. 


\section{Design and development of the core systems}

Interlocking system is one of the core systems for the signaling system. An interlocking system for a typical station layout is firstly designed and developed to ensure a right route establishment. In the interlocking system, the basic interlocking logic relationship among the routes, switches, and signaling must be strictly ensured for the typical station layout. Usually, strict algorithms are used in the core systems to guarantee that conflicting routes can never been established in the same system.

After an interlocking system is designed, it must be tested thoroughly. Based on the station layout, a complete test set should be built. Possibly, a simulation system for the interlocking system is designed to test its logic functions. The test will ensure the correctness and the safety of the system.

Before an interlocking system is designed, the specifications of the system requirements and the system functions should be finalized. The specifications are the basic files of the design and the test. Of course, the typical station layout must be defined to ensure the functions of the interlocking system to be complete.

As the core system, ATP system (one of the on-board system), RBC system, the central control system, and the communication network connecting the core systems should be designed and developed. The processes are the same as the design and development of the interlocking systems. The classification and process can be found in the files of ETCS and CTCS in Ning et al. [3].

In order to design and develop the core system, the prototype for the core system should be designed and developed. Design and development of the prototypes for the core system of the signaling system must obey the design principles of the software engineering. It is divided into the three levels. The first level is the system management level to operate the whole system management including the safety requirement in the vital computer. The second level is to deal with the logic requirements of the systems, i.e. the function rules. The third level is the application level to match function requirements and an application database. Fig. 3 shows the relation of the three levels.

The core systems of a signaling system should include six units based on the four parts. They are the central control unit, the station control unit, the RBC control unit, the on-board control unit, the communication network unit and the wayside unit with radio unit. The six units consist into a minimal signaling

\begin{tabular}{|c|}
\hline $\begin{array}{c}\text { Application Function } \\
\text { (Database) }\end{array}$ \\
\hline Logic Rules \\
\hline Management System \\
\hline
\end{tabular}

Level 3

Level 2

Level 1

Figure 3: Configuration of the software system for a core system. 


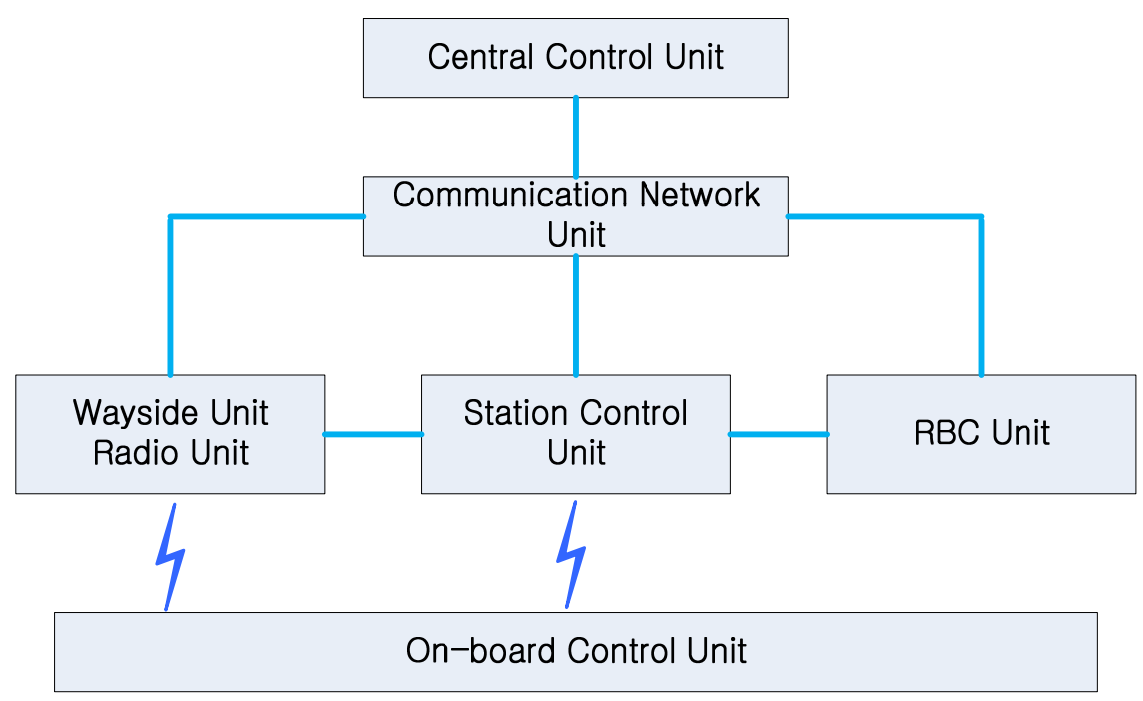

Figure 4: Configuration of a minimal signaling system.

system shown in Fig. 4. The minimal signaling system is the foundation of an application signaling system, and meets all the function requirements of a signaling system. Usually, only the prototype of the minimal signaling system can be found in laboratory.

\section{Re-design of application of the core system for the specified lines}

After development of the core systems for the signaling system, there we have the prototype of the minimal signaling system. Before the application of real signaling system, redesign must be carried out for an applied line based on the core systems and the minimal signaling system. The main task of the redesign is to match the database of a real line and the minimal signaling system with the core systems. The redesign turns the minimal signaling system into a real application signaling system. It needs experts with good skills, while the importance of the step is often ignored. The designers need to know both the core systems and all the requirements of the application line. That is why the signaling system is called as requirements-tailed system, and it costs.

During the redesign, the database for the line and rolling stock must be established. For example, some parameters such as curves and slopes of the line, the parameters for rolling stock, traction features and braking features of rolling stock are needed for the on-board system (ATP or ATO). An interlocking system needs the data for the layout of each station along the lines. There is different number of routes for different station. At each station, the number of switches and the positions of switches are different. For the central control system, all the data from the lines and the requirements are needed to general a train plan and a 
train operation graph. At the same time, the disposition of communication network units and wayside units such as position of design for radio units and balises must be carried out.

In order to simplify the redesign without reducing the correctness, a computer-aid design (CAD) tool is developed. Different CAD tools are designed for the different units, such as Interlocking CAD tool and RBC CAD tool etc in Mitchell [5].

After the redesign of an application signaling system is finished, the whole system is test to verify the functions and safety. To test the signaling system, a simulation system and environments should be built. Test set and test dictionary for a line should been accumulated and established to ensure the test.

\section{Safety assessment}

The signaling system is a system to ensure train operation safety. Therefore, it must be self-safety in its whole life cycle. Fail-safe concept was put forward for the railway signaling system in the early 1900s. Safety assessment for the railway signaling system begins with the start of the system design. From the core system design to redesign of an application signaling system, from the prototype of the core systems to the minimal system, from manufacturing to installation, from operation to maintenance, safety assessment must be taken during the whole life cycle. This is the main reason why a signaling system is complicated and high cost.

There are always two groups of persons in this area. One is to implement the signaling system. Safety assessment is done by another group to ensure the system's implementing to be monitored. Moreover, the second group should involve from the beginning of the system design. In other word, the whole process of the signaling system design, manufacturing, installation and operation must be monitored and assessed. Methods and principles for software engineering must be used for the files management and flow management to do safety assessments of the signaling system. For a big project of railway signaling, the third professional company is invited to do the safety assessment for the project.

What is the meaning for RAMS? The RAMS means Reliability, Availability, Maintainability and Safety of the system. According to EN50126 (CENELEC 1999), the definition of RAMS can be found easily in Theeg and Vlasenko [6]. System reliability is defined as the probability that the system can perform a required function under given conditions for a given time interval. System availability is defined as the ability of a system to be in a state to perform the required function under given conditions over a given time interval, assuming that the required external sources of help is provided. System maintainability is defined as the probability that a given active maintenance action, for a system under given conditions of use, can be carried out within a stated time interval when the maintenance is performed under stated conditions and using stated procedures and resources. System safety is defined as fail-safe requirements that system cannot give dangerous output when a given fault occurs. Reliability and 
maintainability are both probability values which lead to failure and maintenance rate respectively, related to a defined time period. The signaling system is required to be with high availability, i.e. low failure rate and high maintenance rate. System safety is the system quality requirement, and different with reliability. When the concept, as reliability and safety of railway signaling system, is discussed, there are still some of different views as in Ning et al. [2].

In order to ensure the requirements of RAMS for the signaling system to be satisfied, fault-tolerant design, fault-diagnosis and fault test are applied in the design and redesigned for signaling system development. Comparer is often used in the design of signaling system to fulfill the fail-safe requirement of the system. The comparer can be implemented both by hardware and software.

In the safety assessment, a simulation system can also be used to testify if RAMS requirement of the signaling system is performed. It can be used for safety assessment of the core system, the minimal system and the application system of signaling system. Fault set and fault models of signaling system are analyzed and built.

\section{Simulation systems}

Nowadays, it is difficult to imagine how to design and develop a signaling system based on computers without simulation system tools. Simulation systems for the core system and an application system of the railway signaling system have become an important tool for its development, application and maintenance. As far as the functions of the simulation system are concerned, there are many kinds of simulation systems for the signaling system. Some of them have been mentioned in the paper.

Simulation models construction and simulation platforms selection are the first step for development of simulation systems for signaling systems as in Xun et al. [1]. There are numbers of different models and algorithms for the different applications. There are also many kinds of simulation platforms to be selected for development of simulation systems. The above two issues are not addressed in detains here since the limit of the paper contents.

As development tool, a simulation system is developed for the design of core signaling systems and the minimal signaling system. By use of the simulation system, the core signaling systems are designed, and their functions, safety and reliability etc. are tested and proved.

As design tool, a simulation system is developed for redesigning an application signaling system based on the minimal signaling system. The task of the simulation system is to redesign the application signaling system according to the database of the application line. By use of the simulation system, the requirements and configuration of the application system are satisfied, established, and proved.

Usually, a simulation system should be established for an application signaling system. Before the application signaling system is put into operation, the task of the simulation system is to test the functions, safety and fault-tolerant features of the application signaling system and to ensure the correct connection 
among the core signaling systems based on the database of the application line. After the application signaling system is put into operation, the task of the simulation system is to monitor the operation of the application system by sharing the real-time data with the operating system. Meanwhile, as maintenance tool, the simulation system plays an important role in diagnosing a fault and maintaining the system when the fault occurs during the operation of the application system. Moreover, when some of the parts in the application system are revised or upgraded technically, the parts should be tested firstly in the simulation system before be put into the real system.

It can be seen from the above description that the simulation systems have the different classifications and functions. A common databases, test sets and function models should be established and accumulated. The different simulation systems could use the same database, the same test set and the same function models. Interlocking system can be taken as an example. Interlocking function test are the same at the core system development and an application signaling system. As a design tool during the redesign for an application system, it uses the same database with the simulation system of an application signaling system. One of the difficult tasks is to establish a perfect test sets by use of accumulating.

\section{Conclusion}

To get a better understand on the railway signaling system, in the paper, its design and development are defined as the two periods: core system design and application system design, as shown in Fig. 5. It is also introduced in details how simulation systems and safety assessment play an important role in the whole life cycle of a signaling system. The relationship between the phases and systems is

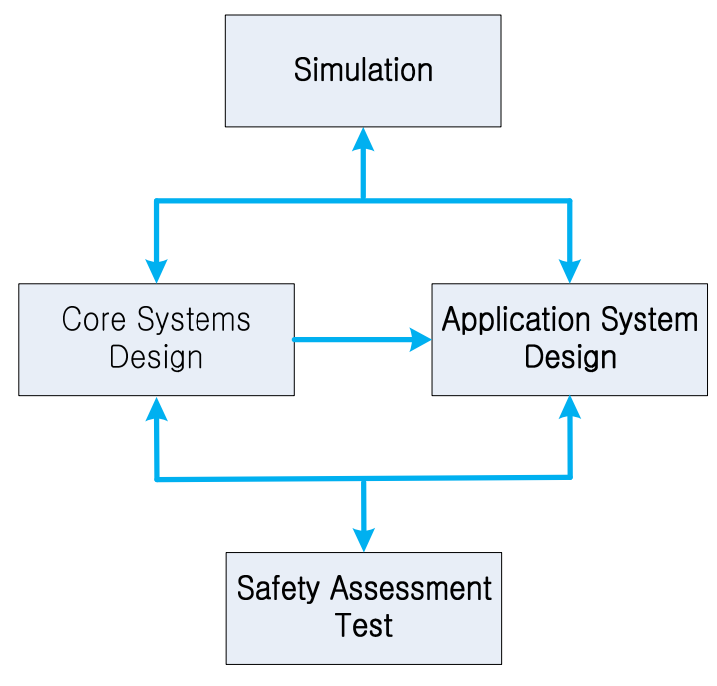

Figure 5: The phases of the signaling system. 
explained. The key points at each phase are described. This paper gives an overall picture and the whole process of railway signaling system. It shows the importance of the railway signaling system.

\section{Acknowledgement}

The research is supported by the National Science foundation: Basic theories and key technologies of train control and organization (60634010).

\section{References}

[1] Xun, J., Ning, B. \& Li, K., Multi-objective optimization method for the ATO system using Cellular Automata. Computers in Railways XI-Computer System Design and Operation in the Railway and Other Transit Systems. vol. 103, eds. Allan, J., Arias, E., Brebbia, C. A., Goodman, C. J., Rumsey, A. F., Sciutto, G. \& Tomii, N., WIT Press: Toledo, pp. 173-182, 2008.

[2] Ning, B., Tang, T., Qiu, K. \& Gao, C., CBTC (Communication Based Train Control): system and development, Computers in Railways X-Computer System Design and Operation in the Railway and Other Transit Systems. vol. 103, eds. Allan, J., Brebbia, C. A., Rumsey, A. F., Sciutto, G., Sone, S. \& Goodman, C. J., WIT Press: Prague, Czech Republic, pp. 413-420, 2006.

[3] Ning, B., Tang, T., Qiu, K., Gao, C. \& Wang, Q., "CTCS-Chinese Train Control System", Computers in Railways IX-Computer System Design and Operation in the Railway and Other Transit Systems. vol. 103, eds. Allan, J., Brebbia, C. A., Hill, R. J., Sciutto, G., \& Sone, S., WIT Press: Dresden, Germany, pp. 262-272, 2004.

[4] Rail Safety and Standards Board, Engineering safety Management (the Yellow Book).

[5] Mitchell, L., The Sustainable Railway Use of Advisory Systems for Energy Savings, IRSE NEWS 151, pp. 2-7, 2009.

[6] Theeg, G. \& Vlasenko, S., Railway Signalling and Interlocking, Eurail Press, pp. 17-21 and pp. 30- 36, 2009. 